\title{
Электрические свойства тонких пленок оксида индия, полученных методом плазменно-термического испарения
}

\author{
(C) А.С. Ильин ${ }^{1}$, А.Н. Мацукатова ${ }^{1}$, П.А. Форш ${ }^{1-3}$, Yu. Vygranenko ${ }^{4}$ \\ ${ }^{1}$ Московский государственный университет им. М.В. Ломоносова (физический фракультет), \\ 119991 Москва, Россия \\ ${ }^{2}$ НИЦ „Курчатовский институт“, \\ 123182 Москва, Россия \\ ${ }^{3}$ Московский физико-технический институт \\ (факультет нано-, био-, информационных и когнитивных технологий), \\ 141700 Долгопрудный, Россия \\ ${ }^{4}$ CTS-UNINOVA, Quinta da Torre, \\ 2829-516 Caparica, Portugal \\ E-mail: as.ilin@physics.msu.ru
}

(Получена 28 февраля 2018 г. Принята к печати 19 марта 2018 г.)

\begin{abstract}
Исследованы структура и электрические свойства прозрачных тонких пленок оксида индия, полученных методом плазменно-термического испарения при различных температурах подложки. Определено, что пленки имеют зернистую структуру. Увеличение температуры подложки приводит к значительному росту проводимости пленок и к уменьшению времени релаксации фотопроводимости. Предложено объяснение влияния температуры подожки на наблюдаемые изменения электрических и фотоэлектрических свойств исследованных пленок оксида индия.
\end{abstract}

DOI: 10.21883/FTP.2018.12.46771.8855

\section{1. Введение}

Тонкие пленки оксида индия находят применение в качестве прозрачных проводящих слоев дисплеев [1] и солнечных элементов [2], на их основе изготавливают транзисторы $[3,4]$ и газовые сенсоры [5-8]. Электрические свойства пленок оксида индия сильно зависят от метода синтеза и структуры. Разработано довольно много методов синтеза пленок оксида индия: золь-гель метод $[9,10]$, электронное и термическое испарение $[4,11,12]$, метод магнетронного напыления [13], осаждение из газовой фазы [14], осаждение с помощью лазерной абляции [15]. Среди многочисленных методов метод термического испарения имеет ряд преимуществ - он сравнительно дешевый, простой, обеспечивает получение пленок в том числе и в промышленных масштабах. Кроме того, этот метод позволяет варьировать структуру, а вместе с тем и электрические параметры пленок (проводимость, концентрацию носителей заряда и их подвижность) в широком диапазоне $[4,12,16]$.

Недавно был разработан метод плазменно-термического напыления тонких пленок оксида индия [17]. Разработанный метод позволяет создавать тонкие прозрачные пленки оксида индия без существенного нагрева подложки в процессе синтеза. Развитие таких методов, позволяющих синтезировать полупроводниковые пленки без нагрева подложки, перспективно для создания электроники на гибких полимерных подложках. В настоящее время много усилий направлено на создание солнечных элементов, светодиодов, транзисторов и фотоприемников на гибких подложках.
В данной работе исследовались электрические и фотоэлектрические свойства тонких пленок оксида индия, полученных методом плазменно-термического напыления при разных температурах подложки. Было определено влияние температуры подложки при синтезе на проводимость пленок оксида индия на постоянном и переменном токе, а также на кинетики фотопроводимости пленок при освещении ультрафиолетовым (УФ) светом.

\section{2. Образцы и методика измерений}

Образцы пленок оксида индия толщиной 100 нм изготавливались методом плазменно-термического испарения (radio-frequency plasma-enhanced reactive thermal evaporation). Процесс изготовления более подробно описан в работе [17]. Были изготовлены 3 партии образцов, отличающихся температурой подложки при осаждении. Оксид индия осаждался при температурах подложки 50 (образец 1), 120 (образец 2) и $150^{\circ} \mathrm{C}$ (образец 3 ).

Структура образцов исследовалась с помощью сканирующего электронного микроскопа Hitachi S-2400. Для проведения электрических и фотоэлектрических измерений на поверхность пленок напылялись алюминиевые контакты. Измерения температурных зависимостей проводимости проводились в области температур $270-470$ K. Исследования импеданса образцов проводились на анализаторе импеданса НР 4192А. Для исследования фотопроводимости образцы освещались светодиодом с максимумом интенсивности вблизи 385 нм. Интенсивность падающего на образец света составляла $5 \mathrm{MBT} / \mathrm{cm}^{2}$. 


\section{3. Экспериментальные результаты}

На рис. 1 представлены изображения образцов 1 и 3, полученные с помощью сканирующей микроскопии. Видно, что образцы имеют зернистую структуру с размером зерен несколько десятков нанометров. При увеличении температуры подложки при синтезе зерна становятся более четко выделенными, кроме того появляются более крупные зерна.

Вольт-амперные характеристики (BAX) исследованных образцов, измеренные на постоянном токе, имеют линейных характер. Проводимость образцов растет с увеличением температуры подложки при синтезе. Значения удельной проводимости образцов 1, 2 и 3 были равны $0.16,2.52$ и $6.9 \mathrm{OM}^{-1} \cdot \mathrm{cm}^{-1}$ соответственно. Значения проводимости пленок довольно высокие, и хотя они меньше проводимости пленок ITO $\left(10^{3} \mathrm{OM}^{-1} \cdot \mathrm{cm}^{-1}[18]\right)$, но приближаются к значению проводимости монокристаллического оксида индия $\left(15 \mathrm{OM}^{-1} \cdot \mathrm{cm}^{-1}[19]\right)$. Учитывая прозрачность пленок в видимом диапазоне [17], такие пленки перспективны для создания проводящих покрытий, осаждаемых при низкой температуре подложки.
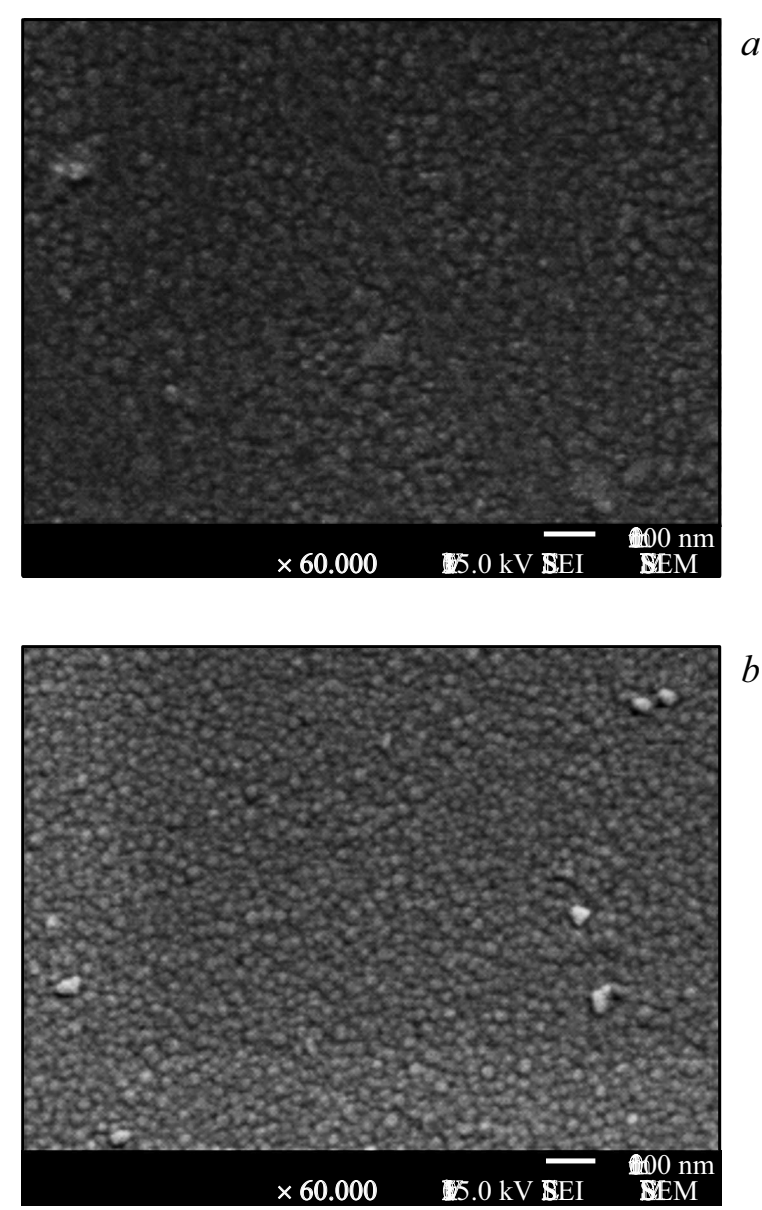

Рис. 1. Изображение образцов $1(a)$ и $2(b)$, полученное с помощью метода сканирующей электронной микроскопии.

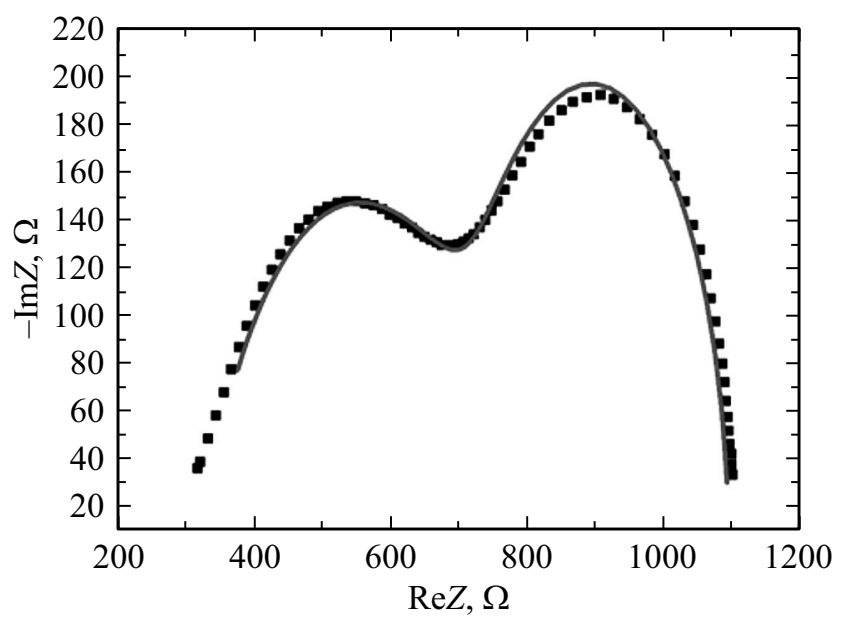

Рис. 2. Годограф импеданса образца 2 (точки) и его аппроксимация (сплошная линия) с использованием предложенной эквивалентной схемы.

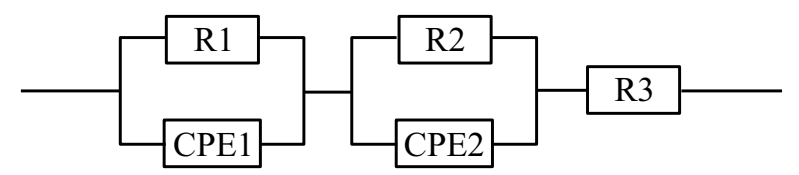

Pис. 3. Эквивалентная схема для исследуемых образцов $\operatorname{In}_{2} \mathrm{O}_{3}$.

С помощью импедансной спектроскопии были построены годографы образцов (зависимости мнимой части импеданса от его действительной части). На рис. 2 для примера показан годограф образца 2. Годографы остальных образцов имеют похожий вид. Полученные годографы хорошо аппроксимируются эквивалентной схемой, изображенной на рис. 3. Данная схема состоит из последовательно соединенных двух R-CPE контуров и резистора. Каждый из R-CPE контуров состоит из параллельно подключённых резистора $\mathrm{R}$ и элемента постоянной фазы CРЕ. Можно предположить, что контур $\mathrm{R}_{1}-\mathrm{CPE}_{1}$ описывает процессы, происходящие в объёме зерен, контур $\mathrm{R}_{2}-\mathrm{CPE}_{2}$ описывает процессы на границе зерен, а сопротивление $\mathrm{R}_{3}$ описывает сопротивление электрических контактов к образцу. Заметим, что элемент СРЕ не имеет точного физического смысла и используется как обобщенный элемент для моделирования импеданса большого спектра различных электрических систем [20]. Импеданс СРЕ-элемента можно записать как

$$
Z_{\mathrm{CPE}}=A^{-1}(i \omega)^{-\alpha},
$$

где $A-$ фактор пропорциональности, $\alpha-$ показатель, который отвечает за частотную зависимость импеданса. Аппроксимированные параметры эквивалентных схем для образцов приведены в таблице. Следует отметить, что для образца 3 наблюдался только один пик на годографе, соответствующий границе зерен, так как максимальной частоты измерительного прибора, по-видимому, недостаточно для регистрации второго пика. В связи 
Значения параметров эквивалентных схем для образцов $\mathrm{In}_{2} \mathrm{O}_{3}$

\begin{tabular}{c|c|c|c|c|c|c|c}
\hline № образца & $\mathrm{R}_{1}$, Ом & $A_{1}, 10^{-10} \mathrm{OM}^{-1} \cdot \Gamma_{Ц^{-\alpha_{1}}}$ & $\alpha_{1}$ & $\mathrm{R}_{2}, \mathrm{OM}_{\mathrm{M}}$ & $A_{2}, 10^{-7} \mathrm{OM}^{-1} \cdot \Gamma_{Ц^{-\alpha_{2}}}$ & $\alpha_{2}$ & $\mathrm{R}_{3}, \mathrm{OM}$ \\
\hline 1 & $1.1 \cdot 10^{4}$ & 4 & 0.65 & $2.4 \cdot 10^{4}$ & 2.6 & 0.60 & 200 \\
2 & 350 & 100 & 1 & 430 & 1.6 & 0.73 & 320 \\
3 & - & - & - & 400 & 2.0 & 0.65 & 150
\end{tabular}

с этим для образца 3 в таблице представлены только значения для контура $\mathrm{R}_{2}-\mathrm{CPE}_{2}$. Видно, что изменение температуры подложки приводит к сильному изменению в параметрах эквивалентной схемы. В частности, с увеличением температуры подложки заметно уменьшаются сопротивления, приписываемые как объему зерен, так и их границам.

Исследования температурных зависимостей проводимости образцов показали, что проводимость практически не зависит от температуры. Это может указывать на то, что исследованные образцы являются вырожденными полупроводниками вследствие сильной нестехиометрии и соответственно большого количества вакансий кислорода, которые являются донорами в оксиде индия.

Также были исследованы процессы кинетики нарастания и спада фотопроводимости. Было установлено, что для всех образцов процессы кинетики нарастания и спада проводимости носят долговременный характер. В качестве примера на рис. 4 приведены зависимости кинетики нарастания и спада фотопроводимости для образца 1. При прекращении освещения образцов УФизлучением значение проводимости в течение долгого времени не возвращается к своему первоначальному значению (т.е. у образцов наблюдается остаточная фотопроводимость). Данный факт является достаточно хорошо известным для нанокристаллических оксидов металлов [21-24]. Объяснение этого процесса связано с адсорбцией и десорбцией кислорода на поверхности оксида.

Полученные зависимости кинетики спада фотопроводимости можно аппроксимировать растянутой экспонентой (функцией Коца):

$$
\sigma_{\mathrm{ph}}(t)=\left(\sigma_{\mathrm{ph}}\right)_{s} \exp \left|-\left(\frac{t}{\tau}\right)^{\beta}\right|,
$$

где $\left(\sigma_{\mathrm{ph}}\right)_{s}-$ стационарная фотопроводимость, $\tau-$ „эффективное“ время релаксации фотопроводимости, $\beta-$ показатель экспоненты. В качестве примера на вставке рис. 4 приведена аппроксимация спада фотопроводимости образца 1. Показатель экспоненты изменяется незначительно от образца к образцу и принимает значение около 0.5. Время релаксации фотопроводимости увеличивается при увеличении температуры подожки от $2.4 \cdot 10^{5}$ с (для образца 1 , осажденного при температуре подожки $50^{\circ} \mathrm{C}$ ) до $6.1 \cdot 10^{5} \mathrm{c}$ (для образца 3 , осажденного при температуре подожки $\left.150^{\circ} \mathrm{C}\right)$. Изменение эффективного времени релаксации фотопроводимости может свидетельствовать об изменении поверхностного

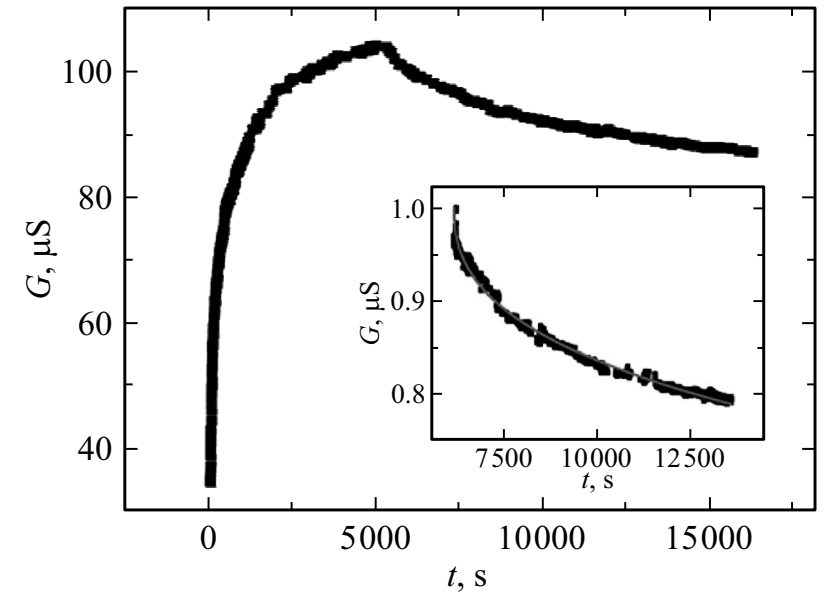

Рис. 4. Зависимости кинетики нарастания и спада фотопроводимости образца 1 при его освещении УФ-светом. На вставке приведен спад фотопроводимости образца и его аппроксимация с помощью растянутой экспоненты.

окружения зерен, а следовательно, и темпа рекомбинации неравновесных носителей заряда, определяющего величину фотопроводимости. Изменение поверхностного покрытия может приводить и к наблюдаемому изменению сопротивления $\mathrm{R}_{2}$ в эквивалентной схеме замещения при изменении температуры подложки.

\section{4. Заключение}

В работе были исследованы электрические и фотоэлектрические свойства тонких пленок оксида индия толщиной 100 нм. Образцы были получены методом плазменно-термического испарения при различных температурах подложки: от 50 до $150^{\circ} \mathrm{C}$. Пленки имели зернистую структуру.

Вольт-амперные характеристики пленок имеют линейный вид, проводимость пленок возрастает более чем на порядок при увеличении температуры подожки до $150^{\circ} \mathrm{C}$ и приближается к значениям проводимости, наблюдающимся на монокристаллическом оксиде индия. В результате измерений импеданса на переменном токе была предложена эквивалентная схема исследованных образцов. Эквивалентная схема учитывает импеданс объема зерен, межзеренных границ и сопротивление электрических контактов. Изменение температуры подложки в процессе осаждения пленок существенным образом изменяет электрические параметры как объема, так и 
границ зерен. В частности, наблюдается резкое уменьшение сопротивления зерен и межзеренных границ при повышении температуры подложки.

Кинетики нарастания и спада фотопроводимости исследованных пленок оксида индия имеют долговременный характер и аппроксимируются растянутой экспонентой. Увеличение температуры подложки при осаждении пленок приводит к увеличению эффективного времени релаксации фотопроводимости. Принимая во внимание тот факт, что, согласно литературным данным, долговременные кинетики фотопроводимости оксида индия связывают с процессами адсорбции и десорбции кислорода на поверхности, был сделан вывод, что при изменении температуры подложки происходит изменение поверхностного окружения зерен в пленке оксида индия, что приводит к изменению темпа рекомбинации неравновесных носителей заряда и изменению времени релаксации фотопроводимости.

Работа выполнена при финансовой поддержке РФФИ (проект № 16-32-60060 мол_а_дк).

\section{Список литературы}

[1] S. Lee, Y. Song, H. Park, A. Zaslavsky, D. Paine. Sol. St. Electron., 135, 94 (2017).

[2] G.G. Untila, T.N. Kost, A.B. Chebotareva. Sol. Energy, 159, 173 (2018).

[3] S. Dellis, I. Isakov, N. Kalfagiannis, K. Tetzner, T. Anthopoulos, D. Koutsogeorgis. J. Mater. Chem. C, 5 (15), 3673 (2017).

[4] Dhananjay, Shiau-Shin Cheng, Chuan-Yi Yang, Chun-Wei Ou, You-Che Chuang, M Chyi Wu, Chih-Wei Chu. J. Phys. D: Appl. Phys., 41 (9), 92006 (2008).

[5] G. Korotcenkov, V. Brinzari, S.H. Han, B.K. Cho. Mater. Chem. Phys., 175, 188 (2016).

[6] A.S. Ilin, M.I. Ikim, P.A. Forsh, T.V. Belysheva, M.N. Martyshov, P.K. Kashkarov, L.I. Trakhtenberg. Sci. Rep., 7 (1), 12204 (2017).

[7] M.N. Martyshov, E.A. Forsh, A.V. Marikutsa, P.A. Forsh, M.N. Rumyantseva, A.M. Gaskov, P.K. Kashkarov. J. Nanoelectron. Optoelectron., 6 (4), 452 (2011).

[8] Е.А. Форш, А.В. Марикуца, М.Н. Мартышов, П.А. Форш, М.Н. Румянцева, А.М. Гаськов, П.К Кашкаров. Рос. нанотехнол., 7 (3-4), 87 (2012). [English version: E.A. Forsh, A.V. Marikutsa, M.N. Martyshov, P.A. Forsh, M.N. Rumyantseva, A.M. Gaskov, P.K. Kashkarov. Nanotechnologies Russ., 7 (3-4), 164 (2012)].

[9] A. Gurlo, M. Ivanovskaya, A. Pfau, U. Weimar, W.G. Spel. Thin Solid Films, 307, 288 (1997).

[10] A. Ilin, M. Martyshov, E. Forsh, P. Forsh, M. Rumyantseva, A. Abakumov, A. Gaskov, P. Kashkarov. Sensors Actuators B Chem., 231 (2), 491 (2016).

[11] J.K. Sheu, Y.K. Su, G.C. Chi, M.J. Jou, C.M. Chang. Appl. Phys. Lett., 72 (25), 3317 (1998).

[12] I.N. Reddy, Ch.V. Reddy, M. Cho, J. Shim, D. Kim. Mater. Res. Express, 4 (8), 86406 (2017).

[13] M. Suchea, N. Katsarakis, S. Christoulakis, S. Nikolopoulou, G. Kiriakidis. Sensors Actuators B Chem., 118, 135 (2006).
[14] S.K. Chong, S.N.A. Azizan, K.W. Chan, H. Nguyen, W.S. Chiu, Z. Aspanut, Ch.F. Dee, S.A. Rahman. NRL, 8 (1), 428 (2013).

[15] C. Li, D. Zhang, S. Han, X. Liu, T. Tang, C. Zhou. Adv. Mater., $15(2), 143$ (2003).

[16] E.A. Forsh, A.V. Marikutsa, M.N. Martyshov, P.A. Forsh, M.N. Rumyantseva, A.M. Gaskov, P.K. Kashkarov. Thin Sol. Films, 558, 320 (2014).

[17] M. Fernandes, Y. Vygranenko, M. Vieira, G. Lavareda, C. Nunes de Carvalho, A. Amaral. Energy Procedia., 102, 96 (2016).

[18] J. Ni, L. Wang, Y. Yang, H. Yan, S. Jin, T.J. Marks, J.R. Ireland, C.R. Kannewurf. Inorg. Chem., 44 (17), 6071 (2005).

[19] J.H.W. de Wit. J. Solid State Chem., 149 (2), 142 (1973).

[20] E. Barsoukov, J.R. Macdonald. Impedance Spectroscopy: Theory, Experiment, and Applications (Hoboken, USA, John Wiley \& Sons, 2005).

[21] Е.А. Форш, А.С. Ильин, М.Н. Мартышов, П.А. Форш, П.К. Кашкаров. Рос. нанотехнол., 9(11-12), 18 (2014). [English version: E.A. Forsh, A.S. Ilyin, M.N. Martyshov, P.A. Forsh, P.K. Kashkarov. Nanotechnologies Russ., 9 (11-12), 618 (2014)].

[22] A.T. Vai, N. Rashidi, Y. Fang, V.L. Kuznetsov, P.P. Edwards. J. Phys. Condens. Matter, 28 (22), 224003 (2016).

[23] A. Dixit, Raghava P. Panguluri, C. Sudakar, P. Kharel, P. Thapa, I. Avrutsky, R. Naik, G. Lawes, B. Nadgorny. Appl. Phys. Lett., 94, 252105 (2009).

[24] E.A. Forsh, A.M. Abakumov, V.B. Zaytsev, E.A. Konstantinova, P.A. Forsh, M.N. Rumyantseva, A.M. Gaskov, P.K. Kashkarov. Thin Sol. Films, 595, 25 (2015).

Редактор А.Н. Смирнов

\section{Electrical properties of indium oxide thin films deposited by plasma-enhanced reactive thermal evaporation}

\author{
A.S. Ilin ${ }^{1}$, A.N. Matsukatova ${ }^{1}$, P.A. Forsh ${ }^{1-3}$, \\ Yu. Vygranenko ${ }^{4}$ \\ ${ }^{1}$ Lomonosov Moscow State University, \\ Faculty of Physics, \\ 119991 Moscow, Russia \\ ${ }^{2}$ NRC Kurchatov Institute, \\ 123182 Moscow, Russia \\ ${ }^{3}$ Moscow Institute of Physics and Technology, \\ Department of Nano-, Bio-, information Technology and \\ Cognitive Science, \\ 141700 Dolgoprudny, Moscow, Russia \\ ${ }^{4}$ CTS-UNINOVA, Quinta da Torre, \\ 2829-516 Caparica, Portugal
}

\begin{abstract}
The structure and electrical properties of transparent indium oxide thin-films deposited by radio-frequency plasmaenhanced reactive thermal evaporation at various substrate temperatures were studied. Granular structure of the films was detected. It has been found that an increase in the substrate temperature leads to significant growth of film conductivity and slow-down of photoconductivity rise and fall processes. An explanation of the influence of substrate temperature on electrical and photoelectrical properties of the indium oxide thin films is also proposed.
\end{abstract}

